\title{
Mitochondria as new therapeutic targets for eradicating cancer stem cells: Quantitative proteomics and functional validation via MCT1/2 inhibition
}

\author{
Rebecca Lamb ${ }^{1,2}$, Hannah Harrison ${ }^{1,2}$, James Hulit',2, Duncan L. Smith³, Michael P. \\ Lisanti ${ }^{1,2}$ and Federica Sotgia ${ }^{1,2}$ \\ ${ }^{1}$ The Manchester Centre for Cellular Metabolism (MCCM), Institute of Cancer Sciences, University of Manchester \\ 2 The Breakthrough Breast Cancer Research Unit, Institute of Cancer Sciences, University of Manchester \\ ${ }^{3}$ The Cancer Research UK Manchester Institute, University of Manchester \\ Correspondence to: Michael P. Lisanti, email: michaelp.lisanti@gmail.com
}

Federica Sotgia, email: fsotgia@gmail.com

Keywords: mitochondrial markers, cancer stem cells, proteomic analysis, ketone metabolism, monocarboxylate transporters (MCTs), AR-C155858, AZD3965, CHCHD2

Received: November 02, $2014 \quad$ Accepted: November 14, $2014 \quad$ Published: November 15, 2014

This is an open-access article distributed under the terms of the Creative Commons Attribution License, which permits unrestricted use, distribution, and reproduction in any medium, provided the original author and source are credited.

\section{ABSTRACT}

Here, we used quantitative proteomics analysis to identify novel therapeutic targets in cancer stem cells and/or progenitor cells. For this purpose, mammospheres from two ER-positive breast cancer cell lines (MCF7 and T47D) were grown in suspension using low-attachment plates and directly compared to attached monolayer cells grown in parallel. This allowed us to identify a subset of proteins that were selectively over-expressed in mammospheres, relative to epithelial monolayers. We focused on mitochondrial proteins, as they appeared to be highly upregulated in both MCF7 and T47D mammospheres. Key mitochondrial-related enzymes involved in betaoxidation and ketone metabolism were significantly upregulated in mammospheres, as well as proteins involved in mitochondrial biogenesis, and specific protein inhibitors of autophagy/mitophagy. Overall, we identified $>\mathbf{4 0}$ "metabolic targets" that were commonly upregulated in both MCF7 and T47D mammospheres. Most of these "metabolic targets" were also transcriptionally upregulated in human breast cancer cells in vivo, validating their clinical relevance. Based on this analysis, we propose that increased mitochondrial biogenesis and decreased mitochondrial degradation could provide a novel mechanism for the accumulation of mitochondrial mass in cancer stem cells. To functionally validate our observations, we utilized a specific MCT1/2 inhibitor (AR-C155858), which blocks the cellular uptake of two types of mitochondrial fuels, namely ketone bodies and L-lactate. Our results indicate that inhibition of MCT1/2 function effectively reduces mammosphere formation, with an IC-50 of $\sim 1 \mu \mathrm{M}$, in both ER-positive and ER-negative breast cancer cell lines. Very similar results were obtained with oligomycin A, an inhibitor of the mitochondrial ATP synthase. Thus, the proliferative clonal expansion of cancer stem cells appears to require oxidative mitochondrial metabolism, related to the re-use of monocarboxylic acids, such as ketones or L-lactate. Our findings have important clinical implications for exploiting mitochondrial metabolism to eradicate cancer stem cells and to prevent recurrence, metastasis and drug resistance in cancer patients. Importantly, a related MCT1/2 inhibitor (AZD3965) is currently in phase I clinical trials in patients with advanced cancers: http://clinicaltrials.gov/show/NCT01791595. 


\section{INTRODUCTION}

Cancer stem cells (CSCs), or tumor-initiating cells (TICs), are thought to be resistant to conventional anticancer therapies, and have been implicated in treatment failure, tumor recurrence and distant metastasis $[1,2]$. Thus, residual treatment-resistant cancer stem cells are believed to be responsible for poor clinical outcomes in most cancer types [2-4]. Since CSCs are relatively rare and elusive, very little is known about them, especially regarding their physiology and metabolic phenotype.

Consistent with the idea that CSCs are resistant to cellular stress, they are able to undergo anchorageindependent growth in low-attachment plates, allowing the formation of 3D spheroids with the properties of cancer stem cells and/or progenitor cells [5]. Under these suspension conditions, most epithelial cancer cells undergo a specialized form of cell death/apoptosis, termed anoikis. Importantly, each of these 3D spheroids is formed from the anchorage-independent clonal expansion of a single cancer stem cell, and not from the self-aggregation of existing cancer cells [5]. Therefore, the preparation of 3D spheroid cultures provides a functional assay to enrich for a population of cells with an epithelial stem cell-like phenotype. In this regard, the behavior of 3D spheroids (also known as mammosphere cultures) prepared from primary breast cancer cells or breast cancer epithelial cell lines are the most well-characterized.

Here, we isolated large numbers of mammospheres from two independent ER-positive breast cancer cell lines, namely MCF7 and T47D cells, in an attempt to better understand their phenotypic behavior at a molecular level. The large-scale preparation of mammospheres allowed us to then perform unbiased label-free proteomics analysis, in an attempt to understand the proteome that is characteristic of cancer stem cells. Interestingly, based on this initial analysis, we noticed that mammospheres dramatically overexpress mitochondrial-related proteins. Thus, we focused on the mitochondrial proteins that were upregulated, relative to cells cultured as epithelial monolayers, in parallel. Based on this analysis, we speculate that CSCs become resistant to stress by fortifying their capacity to produce ATP by oxidative mitochondrial metabolism. Treatment with the MCT1/2 inhibitor (AR$\mathrm{C} 155858$ ) validated that mammosphere formation is dependent on the uptake of specific mitochondrial fuels, such as L-lactate and ketone bodies.

Importantly, over the last several decades, significant progress has been made in understanding the critical role of cellular metabolism in tumor initiation, progression and metastasis, including studies related to ROS production, oxidative stress, glycolysis, glutamine metabolism and oxidative mitochondrial metabolism [6-13]. However, most of these studies have focused on "bulk" cancer cells, but not on cancer stem cells or the progenitor cell population. Thus, there is a great need to understand tumor metabolism in the specific context of "stemness", to identify a metabolic "Achilles' Heel" to eradicate cancer stem cells. As such, our current findings provide an unbiased systematic approach for identifying new metabolic targets in cancer stem cells, using quantitative proteomics analysis, and a strong rationale for therapeutically targeting L-lactate, ketone bodies and mitochondrial metabolism in the cancer stem cell population.

\section{RESULTS}

\section{Proteomic analysis of MCF7 mammospheres}

Monolayer and mammosphere cultures of MCF7 cells, an ER-positive cell line, were subjected to quantitative label-free proteomics analysis. Greater than 500 proteins were found to be upregulated or downregulated. For simplicity, we focused on the proteins that were significantly upregulated in mammospheres, relative to mononlayer cell cultures $(\mathrm{p}<0.05)$. Immediately, we noticed that several mitochondrial proteins were highly upregulated in mammospheres, so we restricted our analysis to mitochondrial proteins and key related metabolic enzymes.

Table 1 shows a non-redundant list of 62 mitochondrial-related proteins that were selectively upregulated in MCF7 mammospheres. Only proteins with a fold increase of $\sim 2$ or greater were selected for this analysis. Note that 9 mitochondrial proteins were infinitely upregulated, as compared with monolayer cultures. A functional analysis of this list revealed that 12 proteins were specifically related to beta-oxidation and ketone metabolism/re-utilization (HSD17B10, BDH1, ACAT1, ACADVL, ACACA, ACLY, HADHB, SUCLG2, ACAD9, HADHA, ECHS1, ACADSB). Also, 8 proteins involved in mitochondrial biogenesis were dramatically upregulated (HSPA9, TIMM8A, GFM1, MRPL45, MRPL17, HSPD1(HSP60), TSFM, TUFM). In addition, many proteins related to electron transport (NDUFB10, COX6B1, PMPCA, COX5B, SDHA, UQCRC1), ATP synthesis (ATP5B, ATPIF1, ATP5A1, ATP5F1, ATP5H, ATP5O), ADP/ATP exchange/transport (SLC25A5), CoQ synthesis (COQ9), or ROS production (GPD2) were also increased. Finally, two proteins involved in the suppression of glycolysis, autophagy and mitophagy were also significantly increased (SOGA1, LRPPRC). Thus, increased mitochondrial biogenesis and decreased mitochondrial degradation could provide a novel mechanism for the overall accumulation of mitochondrial mass in cancer stem cells. 
Table 1: Mitochondrial-related Proteins Up-regulated in MCF7 Mammospheres.

Symbol Gene Description

AK2 Adenylate kinase 2, mitochondrial

ATP5B

GPD2

SOGA1

$\mathrm{CHCHD} 2$

CPOX

HADH2

MCCC1

SLC25A10

HSPA9

TIMM8A

BDH1

ACAT1

NDUFB10

COX6B1

ACADVL

DHTKD1

CCDC47

PGD

ACACA

PC

VDAC3

ALDH4A1

$\mathrm{ECH} 1$

ACLY

GFM1

PMPCA

HADHB

NNT

MRPL45

SUCLG2

LRPPRC

DLST

DLAT

HSPD1

ACAD 9

PTCD3

HARS2

SDHA

ATPIF1

CKMT1/2

$\mathrm{ACO} 2$

COX5B

$\mathrm{MCCC} 2$

CKMT1B

SLC25A1

MRPL17

HADHA

ECHS1

LETM1

TSFM

UQCRC1

ATP5A1

PPA2

COQ9

ATP5F1

SLC25A5

TUFM

KIAA0664

ATP5H

ACADSB

ATP5O

ATP synthase subunit beta

Stress-70 protein, mitochondrial

ATP Citrate Lyase, cytosolic

Mitochondrial elongation factor $\mathrm{G}$

ATPase inhibitor, mitochondrial
Glycerol-3-phosphate dehydrogenase, mitochondrial

Suppressor of glycolysis and autophagy 1

Coiled-coil-helix-coiled-coil-helix domain-containing protein 2 , mitochondrial

Coproporphyrinogen-III oxidase, mitochondrial

Hydroxysteroid (17-Beta) Dehydrogenase 10; HSD17B10 protein

Methylcrotonoyl-CoA carboxylase subunit alpha, mitochondrial

Mitochondrial dicarboxylate carrier

Mitochondrial import inner membrane translocase subunit Tim8 A

D-beta-hydroxybutyrate dehydrogenase, mitochondrial

Acetyl-CoA acetyltransferase, mitochondrial

NADH dehydrogenase [ubiquinone] 1 beta subcomplex subunit 10

Cytochrome c oxidase subunit 6B1

Very-long-chain specific acyl-CoA dehydrogenase, mitochondrial

2-oxoglutarate dehydrogenase E1 component DHKTD1, mitochondrial

Coiled-coil domain-containing protein 47

6-phosphogluconate dehydrogenase (pentose phosphate shunt)

Acetyl-Coenzyme A carboxylase alpha

Pyruvate carboxylase, mitochondrial

Voltage-dependent anion-selective channel protein 3

Delta-1-pyrroline-5-carboxylate dehydrogenase, mitochondrial

Delta(3,5)-Delta(2,4)-dienoyl-CoA isomerase, mitochondrial

Mitochondrial-processing peptidase alpha subunit; paralog is UQCRC2

Mitochondrial trifunctional protein beta subunit

$\mathrm{NAD}(\mathrm{P})$ transhydrogenase, mitochondrial

$39 S$ ribosomal protein $L 45$, mitochondrial

Succinyl-CoA ligase [GDP-forming] subunit beta, mitochondrial

Leucine-rich PPR motif-containing protein, mitochondrial

Dihydrolipoyllysine succinyltransferase, 2-oxoglutarate dehydrogenase

Dihydrolipoyllysine acetyltransferase, pyruvate dehydrogenase complex

$60 \mathrm{kDa}$ heat shock protein, mitochondrial

Acyl-CoA dehydrogenase family member 9 , mitochondrial

Pentatricopeptide repeat-containing protein 3 , mitochondrial

Histidine--tRNA ligase, mitochondrial

Succinate dehydrogenase (ubiquinone) flavoprotein subunit, mitochondrial

Creatine kinase, ubiquitous mitochondrial (EC 2.7.3.2)

Aconitate hydratase, mitochondrial (EC 4.2.1.3)

Cytochrome $\mathrm{c}$ oxidase subunit $5 \mathrm{~B}$, mitochondrial

Methylcrotonoyl-CoA carboxylase beta chain, mitochondrial

Creatine kinase U-type, mitochondrial

Tricarboxylate transport protein, mitochondrial

395 ribosomal protein L17, mitochondrial

Trifunctional enzyme subunit alpha, mitochondrial

Enoyl-CoA hydratase, mitochondrial

LETM1 and EF-hand domain-containing protein 1, mitochondrial

Elongation factor Ts, mitochondrial

Cytochrome b-c1 complex subunit 1, mitochondrial

ATP synthase subunit alpha, mitochondrial

Inorganic pyrophosphatase 2, mitochondrial

Ubiquinone biosynthesis protein COQ9, mitochondrial

ATP synthase, $\mathrm{H}+$ transporting, mitochondrial F0 complex, subunit B1

Solute carrier family 25 (adenine nucleotide translocator), member 5

Elongation factor Tu, mitochondrial

Clustered mitochondria protein homolog

ATP synthase subunit d, mitochondrial

Short/branched chain specific acyl-CoA dehydrogenase, mitochondrial

ATP synthase subunit $O$, mitochondrial
Fold-Upregulation

ANOVA

Infinity

Infinity

Infinity

Infinity

Infinity

Infinity

Infinity

Infinity

Infinity

$298,325.4$

$36,902.6$

$2,592.8$

$1,124.9$

975.80

622.58

573.07

355.26

328.85

292.09

224.71

158.10

136.20

131.96

114.95

100.67

97.22

79.00

60.00

50.38

46.42

32.18

30.92

23.99

23.94

16.44

15.51

14.10

13.05

10.62

10.13

8.32

8.09

7.69

7.54

6.45

5.71

4.80

4.70

4.50

4.16

3.75

3.14

3.05

2.78

2.69

2.51

2.46

2.31

2.31

2.27

2.10

1.92
7.43E-14

3.80E-08

7.80E-13

$5.59 \mathrm{E}-13$

$<1.0 \mathrm{E}-17$

6.70E-12

$1.86 \mathrm{E}-13$

2.80E-12

5.82E-05

2.62E-13

1.45E-11

1.23E-10

1.82E-07

2.22E-09

3.86E-05

3.23E-06

1.11E-07

3.15E-10

4.14E-06

1.40E-09

9.09E-05

2.97E-08

3.93E-05

3.27E-05

7.99E-08

1.32E-05

2.57E-09

7.71E-09

4.20E-10

4.32E-11

6.24E-09

2.63E-12

2.69E-08

$5.07 \mathrm{E}-12$

0.0001

1.90E-10

4.17E-05

2.30E-08

3.74E-11

0.025

4.37E-10

4.79E-11

6.50E-09

2.28E-07

$3.51 \mathrm{E}-06$

$1.22 \mathrm{E}-05$

5.31E-05

1.91E-10

3.33E-07

2.24E-11

0.002

0.002

0.001

0.002

0.001

0.0005

0.002

0.0007

0.0001

6.20E-05

0.004

0.0002

Enzymes in BOLD are related to beta-oxidation and ketone metabolism. 
$15]$.

For comparison purposes, we also performed unbiased label-free proteomic analysis on a second independent ER-positive breast cancer cell line, namely T47D cells.

Our results are summarized in Table 2. Note that 49 mitochondrial-related proteins were specifically over-expressed in T47D mammospheres, as compared with T47D monolayer cultures processed in parallel. Remarkably, 41 of these proteins overlapped with the proteins that were upregulated in MCF7 mammospheres $(41 / 49=84 \%$ overlap $)$. See the Venn diagram presented in Figure 1. Therefore, many of the same biological processes would be expected to be activated or enhanced. Thus, beta-oxidation, ketone re-utilization, mitochondrial biogenesis, and ROS production, with decreased mitochondrial degradation and reduced autophagy, are likely to be key biological features of both MCF7 and T47D mammospheres. Interestingly, CHCHD2 and CPOX were infinitely upregulated in both MCF7 and T47D data sets.

\section{Functional effects of MCT1/2 inhibition on mammosphere formation}

Next, to functionally validate the hypothesis that mammosphere formation may require ketone re-utilization and oxidative mitochondrial metabolism, we used a highly specific inhibitor (AR-C155858) of the relevant monocarboxylate transporters, namely MCT1/2 [14, 15]. $\mathrm{MCT} 1 / 2$ normally function as specific transporters for the uptake of ketone and L-lactate [16]. AR-C155858 effectively inhibits MCT1/2 function, and blocks the cellular uptake of both ketone bodies and L-lactate [14,

\section{Mitochondrial-related Proteins Upregulated in Mammospheres}

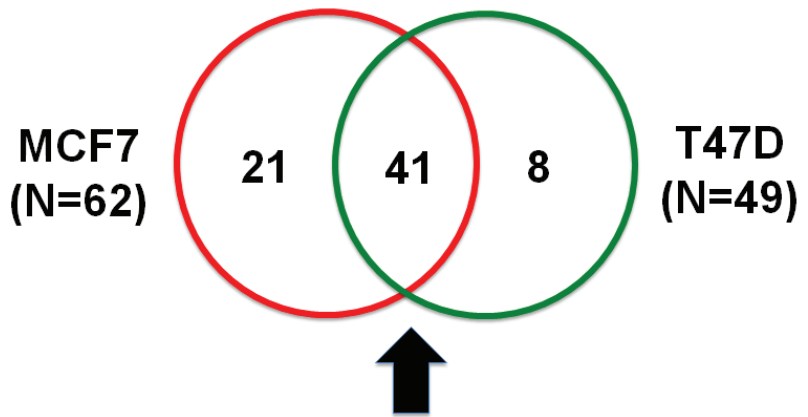

Figure 1: Venn diagram highlighting the conserved upregulation of mitochondrial related proteins in both MCF7 and T47D mammospheres. Note that $>40$ mitochondrial-related proteins were commonly upregulated in both data sets.

Figure 2 shows the effects of increasing concentrations of AR-C155858 on mammosphere formation, using an ER-positive cell line (MCF7). Importantly, the MCT1/2 inhibitor AR-C155858 significantly reduces mammosphere formation, with an IC-50 of $\sim 1 \mu \mathrm{M}$.

As breast cancer stem cells are thought to be ERnegative, we also evaluated the effects of AR-C155858 on an ER-negative cell line, namely MDA-MB-231 cells. Figure 3 shows that the MCT1/2 inhibitor AR-C155858 also effectively reduces mammosphere formation in this cellular context, with an IC-50 of $\sim 1-2 \mu \mathrm{M}$. Therefore, $\mathrm{MCT} 1 / 2$ inhibition may be a new general therapeutic strategy that could be utilized to treat several different epithelial subtypes of human breast cancers.

Thus, 3D spheroid cultures appear to require oxidative mitochondrial metabolism, related to the re-use of monocarboxylic acids (ketones or L-lactate), for the proliferative anchorage-independent expansion of cancer stem cells.

Functional effects of inhibition of the mitochondrial ATP synthase (complex V) on mammosphere formation

Finally, to further validate that mammosphere formation is strictly dependent on oxidative mitochondrial metabolism, we used a well-established investigational compound that potently inhibits the mitochondrial ATP synthase (complex V), namely oligomycin A. Importantly, five protein components of the mitochondrial ATP synthesis were highly up-regulated in MCF7 mammospheres (ATP5B, ATP5A1, ATP5F1, ATP5H, ATP5O).

\section{MCF7 Cell Mammospheres}

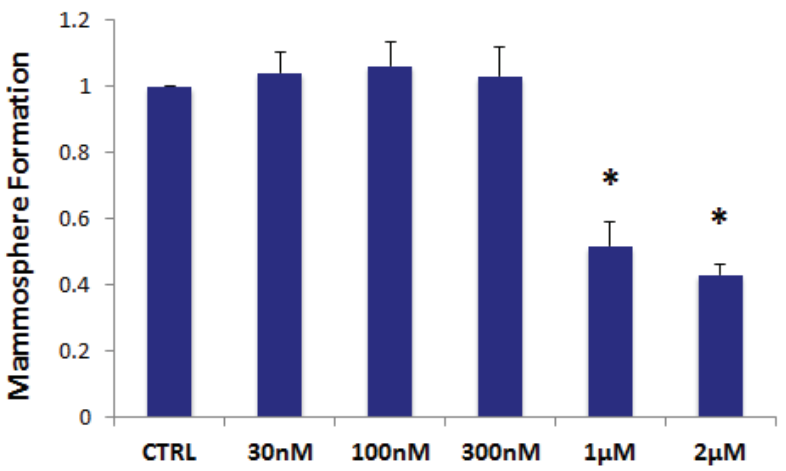

Figure 2: The MCT1/2 inhibitor AR-C155858 significantly reduces mammosphere formation in MCF7 cells. Increasing concentrations of AR-C 155858 inhibit mammosphere formation, using an ER-positive cell line (MCF7). Importantly, AR-C155858 significantly reduces mammosphere formation, with an IC-50 of $\sim 1 \mu \mathrm{M}$. The vehicle-alone control was normalized to one. $(*) \mathrm{p}<6.0 \mathrm{E}-06$. 
Table 2: Mitochondrial-related Proteins Up-regulated in T47D Mammospheres.

\begin{tabular}{|c|c|c|c|}
\hline Symbol & Gene Description & Fold-Upregulation & ANOVA \\
\hline CHCHD2 & Coiled-coil-helix-coiled-coil-helix domain-containing protein 2 , mitochondrial & Infinity & $<1.0 \mathrm{E}-17$ \\
\hline CPOX & Coproporphyrinogen-III oxidase, mitochondrial & Infinity & $<1.0 \mathrm{E}-17$ \\
\hline HSPD1 & $60 \mathrm{kDa}$ heat shock protein, mitochondrial & 69.06 & $1.45 \mathrm{E}-05$ \\
\hline ACADVL & Very-long-chain specific acyl-CoAdehydrogenase, mitochondrial & 66.62 & 2.57E-06 \\
\hline TIMM8A & Mitochondrial import inner membrane translocase subunit Tim8 A & 50.35 & $2.45 \mathrm{E}-06$ \\
\hline ACAT1 & Acetyl-CoA acetyltransferase, mitochondrial & 49.45 & $4.40 \mathrm{E}-10$ \\
\hline HADH2 & Hydroxysteroid (17-Beta) Dehydrogenase 10; HSD17B10 protein & 47.72 & 3.47E-05 \\
\hline HADHB & Mitochondrial trifunctional protein beta subunit & 37.42 & $2.40 \mathrm{E}-12$ \\
\hline ACACA & Acetyl-Coenzyme A carboxylase alpha & 34.85 & 7.27E-11 \\
\hline PGD & 6-phosphogluconate dehydrogenase (pentose phosphate shunt) & 23.93 & 0.03 \\
\hline DLST & Component of 2-oxoglutarate dehydrogenase complex, mitochondrial & 21.39 & 3.19E-14 \\
\hline PDHB & Pyruvate dehydrogenase E1 component subunit beta, mitochondrial & 19.84 & 0.01 \\
\hline MCCC1 & Methylcrotonoyl-CoA carboxylase subunit alpha, mitochondrial & 18.34 & 0.01 \\
\hline DHTKD1 & 2-oxoglutarate dehydrogenase E1 component DHKTD1, mitochondrial & 17.54 & 0.0006 \\
\hline ATPIF1 & ATPase inhibitor, mitochondrial & 16.36 & 0.01 \\
\hline PMPCA & Mitochondrial-processing peptidase alpha subunit; paralog of UQCRC2 & 15.96 & 1.83E-13 \\
\hline BDH1 & D-beta-hydroxybutyrate dehydrogenase, mitochondrial & 15.17 & 0.0003 \\
\hline SLC25A10 & Mitochondrial dicarboxylate carrier & 13.81 & 0.015 \\
\hline VDAC3 & Voltage-dependent anion-selective channel protein 3 & 12.91 & 1.11E-08 \\
\hline MRPL47 & 39S ribosomal protein L47, mitochondrial & 10.69 & 0.03 \\
\hline ECH1 & Delta(3,5)-Delta(2,4)-dienoyl-CoA isomerase, mitochondrial & 10.49 & 8.18E-08 \\
\hline MCCC2 & Methylcrotonoyl-CoA carboxylase beta chain, mitochondrial & 10.38 & $1.52 \mathrm{E}-06$ \\
\hline NNT & $\mathrm{NAD}(\mathrm{P})$ transhydrogenase, mitochondrial & 10.08 & 4.46E-10 \\
\hline ALDH4A1 & Delta-1-pyrroline-5-carboxylate dehydrogenase, mitochondrial & 8.18 & 9.73E-05 \\
\hline LRPPRC & Leucine-rich PPR motif-containing protein, mitochondrial & 8.17 & $6.62 \mathrm{E}-10$ \\
\hline SUCLG2 & Succinyl-CoA ligase [GDP-forming] subunit beta, mitochondrial & 7.54 & $3.10 \mathrm{E}-06$ \\
\hline ACLY & ATP Citrate Lyase, cytosolic & 7.01 & 0.01 \\
\hline CKMT1/2 & Creatine kinase, ubiquitous mitochondrial & 6.81 & 4.73E-11 \\
\hline MRPL45 & $39 S$ ribosomal protein L45, mitochondrial & 6.02 & $9.00 \mathrm{E}-11$ \\
\hline GPD2 & Glycerol-3-phosphate dehydrogenase, mitochondrial & 5.99 & 0.002 \\
\hline C21orf33 & ES1 protein homolog, mitochondrial & 5.97 & 0.005 \\
\hline HARS2 & Probable histidine--tRNA ligase, mitochondrial & 5.82 & 2.03E-07 \\
\hline PTCD3 & Pentatricopeptide repeat-containing protein 3 , mitochondrial & 5.57 & 0.01 \\
\hline SQRDL & Sulfide:quinone oxidoreductase, mitochondrial & 4.94 & 1.15E-08 \\
\hline ATP5F1 & ATP synthase, $\mathrm{H}+$ transporting, mitochondrial F0 complex, subunit B1 & 4.38 & 0.0005 \\
\hline DLAT & Dihydrolipoyllysine acetyltransferase, pyruvate dehydrogenase complex & 4.31 & $6.71 \mathrm{E}-09$ \\
\hline HSPA9 & Stress-70 protein, mitochondrial & 4.14 & 0.03 \\
\hline LETM1 & LETM1 and EF-hand domain-containing protein 1, mitochondrial & 4.14 & 0.01 \\
\hline PC & Pyruvate carboxylase, mitochondrial & 3.39 & 1.33E-05 \\
\hline MRPL17 & $39 S$ ribosomal protein $L 17$, mitochondrial & 3.30 & 0.01 \\
\hline SLC25A1 & Tricarboxylate transport protein, mitochondrial & 3.22 & 0.03 \\
\hline PDCD8 & Apoptosis-inducing factor 1 , mitochondrial & 3.22 & 5.92E-06 \\
\hline UQCRC1 & Cytochrome b-c1 complex subunit 1 , mitochondrial & 2.94 & 1.16E-05 \\
\hline SOGA1 & Suppressor of glycolysis and autophagy 1 & 2.90 & $1.01 \mathrm{E}-05$ \\
\hline CKMT1A & Creatine kinase U-type, mitochondrial & 2.90 & $5.42 \mathrm{E}-05$ \\
\hline CKMT1B & Creatine kinase U-type, mitochondrial & 2.66 & $5.43 \mathrm{E}-05$ \\
\hline CPT1A & Carnitine palmitoyltransferase $1 \mathrm{~A}$, mitochondrial protein & 2.51 & 0.0005 \\
\hline ACADS & Medium-chain specific acyl-CoA dehydrogenase, mitochondrial (EC 1.3.99.3) & 2.47 & 0.0001 \\
\hline MRPS22 & $28 \mathrm{~S}$ ribosomal protein S22, mitochondrial & 2.12 & 0.0001 \\
\hline
\end{tabular}

Proteins shown in BOLD were also up-regulated in MCF7 Mammospheres.

Figure 4A,B shows the effects of increasing concentrations of oligomycin A on mammosphere formation. Note that oligomycin A significantly reduces mammosphere formation in MCF7 cells, with an IC-50 of $100 \mathrm{nM}$. Oligomycin A also significantly inhibited mammosphere formation in MDA-MB-231 cells, but with less potency, with an IC-50 between 5-10 $\mu \mathrm{M}$. As such, oligomycin A was 50-100 times less potent in MDAMB-231 cells, as compared with MCF7 mammospheres.

Therefore, MCT1/2 inhibition may be a more effective strategy for eradicating cancer stem cells in multiple breast cancer types, rather than targeting the mitochondrial ATP synthase.

\section{Clinical relevance of mitochondrial targets in human breast cancers}

To determine the potential clinical relevance of our findings, we next assessed whether the "metabolic targets" that we identified in mammospheres were also 
Table 3: "Metabolic Targets" Over-Expressed in Mammospheres are also Transcriptionally Up-regulated in Human Breast Cancer Cells In Vivo (Cancer Epithelia vs. Tumor Stroma).

\begin{tabular}{|c|c|c|c|}
\hline Symbol & Gene Description & $\begin{array}{l}\text { Fold-Upregulation } \\
\text { (Epithelial/Stromal) }\end{array}$ & P-value \\
\hline $\mathrm{CHCHD2}$ & Coiled-coil-helix-coiled-coil-helix domain-containing protein 2, mitochondrial & 5.79 & 1.85E-07 \\
\hline ACACA & Acetyl-Coenzyme A carboxylase alpha & 5.59 & 3.89E-07 \\
\hline MCCC2 & Methylcrotonoyl-CoA carboxylase beta chain, mitochondrial & 5.48 & $5.78 \mathrm{E}-07$ \\
\hline ATP5F1 & ATP synthase, $\mathrm{H}+$ transporting, mitochondrial F0 complex, subunit B1 & 5.39 & 7.83E-07 \\
\hline ATP50 & ATP synthase subunit $O$, mitochondrial & 5.12 & 2.13E-06 \\
\hline ATP5B & ATP synthase subunit beta, mitochondrial & 5.04 & $2.75 \mathrm{E}-06$ \\
\hline COX5B & Cytochrome $\mathrm{c}$ oxidase subunit $5 \mathrm{~B}$, mitochondrial & 5.03 & 2.86E-06 \\
\hline ATP5A1 & ATP synthase subunit alpha, mitochondrial & 5.01 & 3.09E-06 \\
\hline PDHB & Pyruvate dehydrogenase E1 component subunit beta, mitochondrial & 4.51 & 1.75E-05 \\
\hline LRPPRC & Leucine-rich PPR motif-containing protein, mitochondrial & 4.34 & 3.15E-05 \\
\hline ECHS1 & Enoyl-CoA hydratase, mitochondrial & 4.05 & $8.22 \mathrm{E}-05$ \\
\hline ATP5H & ATP synthase subunit $d$, mitochondrial & 4.01 & $9.48 \mathrm{E}-05$ \\
\hline VDAC3 & Voltage-dependent anion-selective channel protein 3 & 3.94 & 1.19E-04 \\
\hline HSPA9 & Stress-70 protein, mitochondrial & 3.69 & 2.64E-04 \\
\hline ATPIF1 & ATPase inhibitor, mitochondrial & 3.60 & $3.48 \mathrm{E}-04$ \\
\hline SLC25A5 & Solute carrier family 25 (adenine nucleotide translocator), member 5 & 3.49 & 4.81E-04 \\
\hline ACLY & ATP Citrate Lyase, cytosolic & 3.48 & 4.97E-04 \\
\hline HSPD1 & $60 \mathrm{kDa}$ heat shock protein, mitochondrial & 3.42 & $5.93 \mathrm{E}-04$ \\
\hline TUFM & Elongation factor Tu, mitochondrial & 3.38 & 6.74E-04 \\
\hline C21orf33 & ES1 protein homolog, mitochondrial & 3.31 & $8.40 \mathrm{E}-04$ \\
\hline HADHA & Trifunctional enzyme subunit alpha, mitochondrial & 3.27 & $9.34 \mathrm{E}-04$ \\
\hline MRPS22 & $28 S$ ribosomal protein $\mathrm{S} 22$, mitochondrial & 3.27 & $9.31 \mathrm{E}-04$ \\
\hline HADH2 & Hydroxysteroid (17-Beta) Dehydrogenase 10; HSD17B10 protein & 3.22 & 1.10E-03 \\
\hline PPA2 & Inorganic pyrophosphatase 2, mitochondrial & 3.19 & 1.17E-03 \\
\hline SQRDL & Sulfide:quinone oxidoreductase, mitochondrial & 3.14 & $1.38 \mathrm{E}-03$ \\
\hline $\begin{array}{l}\text { HADHB } \\
\text { SUCLG2 }\end{array}$ & $\begin{array}{l}\text { Mitochondrial trifunctional protein beta subunit } \\
\text { Succinyl-CoA ligase [GDP-forming] subunit beta, mitochondrial }\end{array}$ & $\begin{array}{l}3.06 \\
3.03\end{array}$ & $\begin{array}{l}1.73 \mathrm{E}-03 \\
1.89 \mathrm{E}-03\end{array}$ \\
\hline PTCD3 & Pentatricopeptide repeat-containing protein 3 , mitochondrial & 2.98 & $2.15 \mathrm{E}-03$ \\
\hline Cox6B1 & Cytochrome $\mathrm{c}$ oxidase subunit $6 \mathrm{~B} 1$ & 2.97 & 2.21E-03 \\
\hline MRPL17 & $39 S$ ribosomal protein L17, mitochondrial & 2.94 & $2.38 \mathrm{E}-03$ \\
\hline LETM1 & LETM1 and EF-hand domain-containing protein 1, mitochondrial & 2.81 & $3.45 \mathrm{E}-03$ \\
\hline $\operatorname{CCDC} 47$ & Coiled-coil domain-containing protein 47 & 2.70 & $4.68 \mathrm{E}-03$ \\
\hline DLAT & Dihydrolipoyllysine acetyltransferase, pyruvate dehydrogenase complex & 2.63 & $5.53 \mathrm{E}-03$ \\
\hline MCcC1 & Methylcrotonoyl-CoA carboxylase subunit alpha, mitochondrial & 2.40 & $9.99 \mathrm{E}-03$ \\
\hline AK2 & Adenylate kinase 2 , mitochondrial & 2.20 & $1.59 \mathrm{E}-02$ \\
\hline ECH1 & Delta(3,5)-Delta(2,4)-dienoyl-CoA isomerase, mitochondrial & 1.97 & 2.72E-02 \\
\hline ACAT1 & Acetyl-CoA acetyltransferase, mitochondrial & 1.93 & 2.97E-02 \\
\hline BDH1 & D-beta-hydroxybutyrate dehydrogenase, mitochondrial & 1.86 & 3.45E-02 \\
\hline ACO2 & Aconitate hydratase, mitochondrial (EC 4.2.1.3) & 1.83 & 3.64E-02 \\
\hline
\end{tabular}

-Transcriptional profiling data derived from the analysis of $\mathrm{N}=\mathbf{2 8}$ breast cancer patients are shown, high-lighting the levels of foldupregulation observed in the epithelial cancer cell compartment (relative to the tumor stroma), and corresponding $p$-values derived from the analysis of these clinical samples.

-Proteins listed above (39 in total) were all upregulated either in MCF7 or T47D mammospheres (See Tables 1 \& 2).

-Proteins shown in BOLD were commonly upregulated in both MCF7 and T47D mammospheres (21 out of 39 proteins).

transcriptionally upregulated in human breast cancer cells in vivo.

For this purpose, we employed a published clinical data set of $\mathrm{N}=28$ breast cancer patients in which their tumor samples were subjected to laser-capture microdissection, to physically separate epithelial cancer cells from their adjacent tumor stroma [17]. Table 3 presents a summary of these findings. Overall, 39 of the "metabolic targets" that we identified in mammospheres (Tables 1 \& 2) were also transcriptionally elevated in human breast cancer cells in vivo (Table 3 ) and the majority of these targets were upregulated in both MCF7 and T47D mammospheres (21 out of $39, \sim 54 \%$ ).

In light of these findings, the new "metabolic targets" that we identified in mammospheres are especially clinically relevant, for improving both the diagnosis and treatment of human breast cancers.

\section{DISCUSSION}

Previous immuno-histochemical studies have shown that markers of cell proliferation (Ki67) and mitochondrial mass/function (TOMM20 and Complex IV activity) specifically co-localize to the basal stem cell layer in human oral mucosal tissue [18]. Interestingly, in this context, MCT1 was the most specific marker of the basal stem cell layer, suggesting that normal stem cells may use L-lactate and ketone bodies to fuel oxidative 
mitochondrial metabolism and stem cell proliferation [18]. Similarly, Ki67, mitochondrial markers and MCT1 also co-localized in aggressive head and neck tumor cells, consistent with the idea that amplification of mitochondrial metabolism may contribute to human tumor formation and cancer progression [18].

Numerous studies have also implicated ketone bodies and L-lactate metabolism in cancer biology and/or "stemness" in cancer cells. For example, treatment with mitochondrial fuels (such as L-lactate and 3-hydroxybutyrate) is sufficient to stimulate mitochondrial biogenesis in MCF7 cells and dramatically increases the levels of gene transcripts normally expressed in embryonic, neuronal and hematopoietic stem cells [19]. Importantly, the transcriptional profiles of lactate- or ketone-treated MCF7 cells effectively predicted poor clinical outcome (tumor recurrence and metastasis) in ER-positive breast cancer patients [19]. Consistent with these findings, Cuyas et al. recently showed that cancer stem cells created by silencing of E-cadherin expression prefer to use L-lactate and ketone bodies as mitochondrial fuels [20].

Here, using unbiased label-free proteomics analysis, we show that mammospheres (a population of cells enriched in cancer stem cells and progenitor cells) functionally overexpress numerous mitochondrial proteins, related to mitochondrial biogenesis, electron transport, OXPHOS, ATP synthesis, as well as betaoxidation and ketone re-utilization. The potential clinical relevance of these targets was further validated using a previously published data set of human breast cancer samples ( $\mathrm{N}=28$ patients), that were subjected to lasercapture microdissection, to separate the epithelial tumor cells from the adjacent tumor stroma [17]. Thus, these novel mitochondrial-based targets may reveal a metabolic "Achilles' Heel" to allow the eradication of cancer stem

\section{MDA-MB-231 Cell Mammospheres}

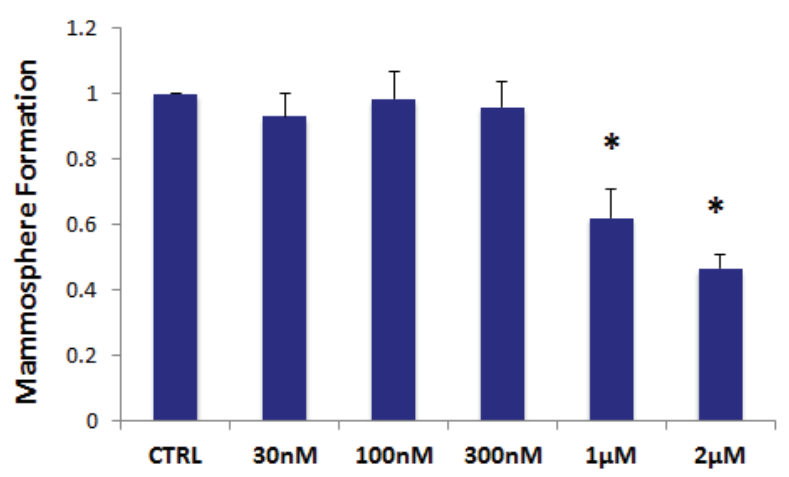

Figure 3: The MCT1/2 inhibitor AR-C155858 significantly reduces mammosphere formation in MDA-MB-231 cells. Note that AR-C155858 also effectively reduces mammosphere formation in this cellular context, with an IC-50 of $\sim 1-2 \mu \mathrm{M}$. The vehicle-alone control was normalized to one. $(*) \mathrm{p}<0.0005$. cells.

In accordance with this idea, we demonstrate that therapeutic targeting of MCT1/2 in cancer stem cells may be a viable strategy, via inhibiting the uptake of necessary key mitochondrial fuels (ketone bodies and L-lactate), that may be required for anchorage-independent growth, as well as cancer stem cell proliferative expansion and survival. Further validation was also provided by experiments with oligomycin A, a well-established inhibitor of the mitochondrial ATP synthase (complex V).

Interestingly, here we observed that the mitochondrial protein CHCHD2 was infinitely upregulated in both MCF7 and T47D mammospheres (Tables 1 and 2), and was also the most highly transcriptionally upregulated protein in the context of human breast cancer cells in vivo (Table 3). Thus, future studies may be warranted on the specific role of CHCHD2 in cancer stem cell metabolism. Currently, very little is known about CHCHD2. However, CHCHD2 has been previously implicated functionally in the response to hypoxia and in the transcriptional upregulation of members of the OXPHOS complexes, as well as a positive regulator of cell migration [21-23].

In conclusion, based on our quantitative proteomics analysis and functional validation studies using

A

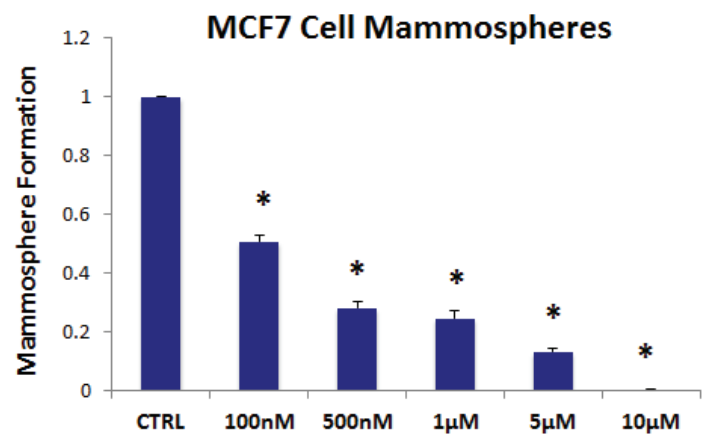

B

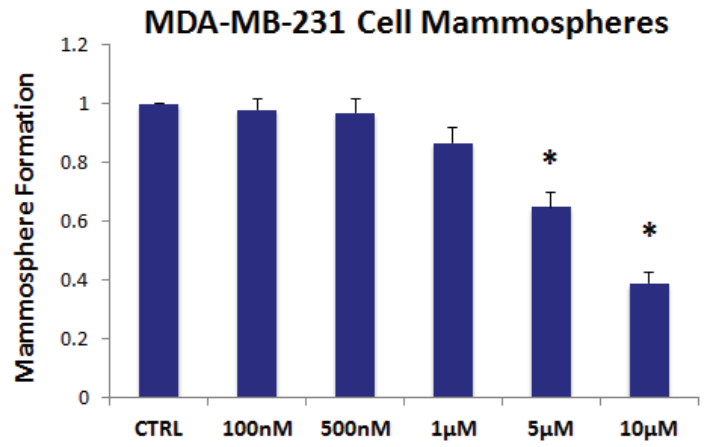

Figure 4: The mitochondrial ATP synthase inhibitor oligomycin A significantly reduces mammosphere formation in both MCF7 and MDA-MB-231 cells. Note that oligomycin A effectively reduces mammosphere formation, with an IC-50 of $\sim 100 \mathrm{nM}$ in MCF7 cells (panel A) and $\sim 5-10$ $\mu \mathrm{M}$ in MDA-MB-231 cells (panel B). Thus, oligomycin A was nearly 50-100 times more potent in MCF7 cells. The vehiclealone control was normalized to one. $\left({ }^{*}\right) \mathrm{p}<3.2 \mathrm{E}-06$. 
mammosphere cultures, we propose that mitochondria are new therapeutic targets for eradicating cancer stem cells, to prevent tumor recurrence, metastasis and poor clinical outcome in breast cancer patients.

\section{MATERIALS AND METHODS}

\section{Materials}

Breast cancer cell lines (MCF7, T47D and MDAMB-231 cells) were purchased from the ATCC. ARC155858 was obtained commercially from MedChem Express (UK). Gibco-brand cell culture media (DMEM/ F12) was purchased from Life Technologies. Oligomycin A was obtained from Sigma-Aldrich.

\section{Mammosphere Culture}

A single cell suspension was prepared using enzymatic (1x Trypsin-EDTA, Sigma Aldrich, \#T3924), and manual disaggregation (25 gauge needle) to create a single cell suspension [5]. Cells were plated at a density of 500 cells $/ \mathrm{cm} 2$ in mammosphere medium (DMEM-F12/ B27/20ng/ml EGF/PenStrep) in non-adherent conditions, in culture dishes coated with (2-hydroxyethylmethacrylate) (poly-HEMA, Sigma, \#P3932). Cells were grown for 5 days and maintained in a humidified incubator at $37^{\circ} \mathrm{C}$ at an atmospheric pressure in $5 \%(\mathrm{v} / \mathrm{v})$ carbon dioxide/air. After 5 days for culture, spheres $>50 \mu \mathrm{m}$ were counted using an eye piece graticule, and the percentage of cells plated which formed spheres was calculated and is referred to as percentage mammosphere formation, and was normalized to one $(1=100 \% \mathrm{MSF})$. For proteomic analysis, mammospheres were collected by centrifugation at $800 \mathrm{rpm}$ for 10 minutes.

\section{Label-free Quantitative Proteomics analysis}

Cell lysates were prepared for trypsin digestion by sequential reduction of disulphide bonds with TCEP and alkylation with MMTS [24]. Then, the peptides were extracted and prepared for LC-MS/MS. All LC-MS/MS analyses were performed on an LTQ Orbitrap XL mass spectrometer (Thermo Scientific, San Jose, CA) coupled to an Ultimate 3000 RSLCnano system (Thermo Scientific, formerly Dionex, The Netherlands). Xcalibur raw data files acquired on the LTQ-Orbitrap XL were directly imported into Progenesis LCMS software (Waters Corp., Milford, MA, formerly Non-linear dynamics, Newcastle upon Tyne, UK) for peak detection and alignment. Data were analyzed using the Mascot search engine. Five replicates were analyzed for each sample type $(\mathrm{N}=5)$. Statistical analyses were performed using ANOVA and only fold-changes in proteins with a p-value less than 0.05 were considered significant.

A more detailed proteomics protocol is provided as Supplementary Information.

\section{Data Mining}

To firmly establish the clinical relevance of our results from the quantitative proteomics analysis of mammosheres, we re-analyzed the transcriptional profiles of epithelial breast cancer cells and adjacent tumor stromal cells that were physically separated by laser-capture microdissection (from $\mathrm{N}=28$ human breast cancer patients) [17].

\section{ACKNOWLEDGEMENTS}

We thank the University of Manchester for providing start-up funds that contributed to the success of this study. RL, HH, MPL, and FS were supported, in part, by funding from the European Union (ERC Advanced Grant), Breakthrough Breast Cancer, and the Manchester Cancer Research Centre (MCRC). DLS was core-funded by CRUK.

\section{REFERENCES}

1. Zhang M and Rosen JM. Stem cells in the etiology and treatment of cancer. Curr Opin Genet Dev. 2006; 16(1):6064.

2. Chandler JM and Lagasse E. Cancerous stem cells: deviant stem cells with cancer-causing misbehavior. Stem Cell Res Ther. 2010; 1(2):13.

3. Scopelliti A, Cammareri P, Catalano V, Saladino V, Todaro M and Stassi G. Therapeutic implications of Cancer Initiating Cells. Expert Opin Biol Ther. 2009; 9(8):10051016.

4. Duggal R, Minev B, Geissinger U, Wang H, Chen NG, Koka PS and Szalay AA. Biotherapeutic approaches to target cancer stem cells. J Stem Cells. 2013; 8(3-4):135149.

5. Shaw FL, Harrison H, Spence K, Ablett MP, Simoes BM, Farnie $\mathrm{G}$ and Clarke RB. A detailed mammosphere assay protocol for the quantification of breast stem cell activity. $\mathrm{J}$ Mammary Gland Biol Neoplasia. 2012; 17(2):111-117.

6. Borodkina A, Shatrova A, Abushik P, Nikolsky N and Burova E. Interaction between ROS dependent DNA damage, mitochondria and p38 MAPK underlies senescence of human adult stem cells. Aging (Albany NY). 2014; 6(6):481-495.

7. Marcinek DJ and Siegel MP. Targeting redox biology to reverse mitochondrial dysfunction. Aging (Albany NY). 2013; 5(8):588-589.

8. Yoon MJ, Lee AR, Jeong SA, Kim YS, Kim JY, Kwon 
YJ and Choi KS. Release of Ca2+ from the endoplasmic reticulum and its subsequent influx into mitochondria trigger celastrol-induced paraptosis in cancer cells. Oncotarget. 2014; 5(16):6816-6831.

9. Corazao-Rozas P, Guerreschi P, Jendoubi M, Andre F, Jonneaux A, Scalbert C, Garcon G, Malet-Martino M, Balayssac S, Rocchi S, Savina A, Formstecher P, Mortier L, Kluza J and Marchetti P. Mitochondrial oxidative stress is the Achille's heel of melanoma cells resistant to Brafmutant inhibitor. Oncotarget. 2013; 4(11):1986-1998.

10. Hall A, Meyle KD, Lange MK, Klima M, Sanderhoff M, Dahl C, Abildgaard C, Thorup K, Moghimi SM, Jensen PB, Bartek J, Guldberg P and Christensen C. Dysfunctional oxidative phosphorylation makes malignant melanoma cells addicted to glycolysis driven by the (V600E)BRAF oncogene. Oncotarget. 2013; 4(4):584-599.

11. Muppani N, Nyman U and Joseph B. TAp73alpha protects small cell lung carcinoma cells from caspase-2 induced mitochondrial mediated apoptotic cell death. Oncotarget. 2011; 2(12):1145-1154.

12. Francipane $\mathrm{MG}$ and Lagasse E. Selective targeting of human colon cancer stem-like cells by the mTOR inhibitor Torin-1. Oncotarget. 2013; 4(11):1948-1962.

13. Cufi S, Corominas-Faja B, Vazquez-Martin A, OliverasFerraros C, Dorca J, Bosch-Barrera J, Martin-Castillo B and Menendez JA. Metformin-induced preferential killing of breast cancer initiating CD44+CD24-/low cells is sufficient to overcome primary resistance to trastuzumab in HER2+ human breast cancer xenografts. Oncotarget. 2012; 3(4):395-398.

14. Ovens MJ, Manoharan C, Wilson MC, Murray CM and Halestrap AP. The inhibition of monocarboxylate transporter 2 (MCT2) by AR-C155858 is modulated by the associated ancillary protein. Biochem J. 2010; 431(2):217225.

15. Ovens MJ, Davies AJ, Wilson MC, Murray CM and Halestrap AP. AR-C155858 is a potent inhibitor of monocarboxylate transporters MCT1 and MCT2 that binds to an intracellular site involving transmembrane helices 7-10. Biochem J. 2010; 425(3):523-530.

16. Draoui N, Schicke O, Seront E, Bouzin C, Sonveaux $\mathrm{P}$, Riant $\mathrm{O}$ and Feron $\mathrm{O}$. Antitumor activity of 7-aminocarboxycoumarin derivatives, a new class of potent inhibitors of lactate influx but not efflux. Mol Cancer Ther. 2014; 13(6):1410-1418.

17. Casey T, Bond J, Tighe S, Hunter T, Lintault L, Patel O, Eneman J, Crocker A, White J, Tessitore J, Stanley M, Harlow S, Weaver D, Muss H and Plaut K. Molecular signatures suggest a major role for stromal cells in development of invasive breast cancer. Breast Cancer Res Treat. 2009; 114(1):47-62.

18. Curry JM, Tuluc M, Whitaker-Menezes D, Ames JA, Anantharaman A, Butera A, Leiby B, Cognetti DM, Sotgia F, Lisanti MP and Martinez-Outschoorn UE. Cancer metabolism, stemness and tumor recurrence: MCT1 and
MCT4 are functional biomarkers of metabolic symbiosis in head and neck cancer. Cell Cycle. 2013; 12(9):1371-1384.

19. Martinez-Outschoorn UE, Prisco M, Ertel A, Tsirigos A, Lin Z, Pavlides S, Wang C, Flomenberg N, Knudsen ES, Howell A, Pestell RG, Sotgia F and Lisanti MP. Ketones and lactate increase cancer cell "stemness," driving recurrence, metastasis and poor clinical outcome in breast cancer: achieving personalized medicine via MetaboloGenomics. Cell Cycle. 2011; 10(8):1271-1286.

20. Cuyas E, Corominas-Faja B and Menendez JA. The nutritional phenome of EMT-induced cancer stem-like cells. Oncotarget. 2014; 5(12):3970-3982.

21. Aras S, Bai M, Lee I, Springett R, Huttemann M and Grossman LI. MNRR1 (formerly CHCHD2) is a biorganellar regulator of mitochondrial metabolism. Mitochondrion. 2014.

22. Aras S, Pak O, Sommer N, Finley R, Jr., Huttemann M, Weissmann $\mathrm{N}$ and Grossman LI. Oxygen-dependent expression of cytochrome c oxidase subunit 4-2 gene expression is mediated by transcription factors RBPJ, CXXC5 and CHCHD2. Nucleic Acids Res. 2013; 41(4):2255-2266.

23. Seo M, Lee WH and Suk K. Identification of novel cell migration-promoting genes by a functional genetic screen. Faseb J. 2010; 24(2):464-478.

24. Holland M, Castro FV, Alexander S, Smith D, Liu J, Walker M, Bitton D, Mulryan K, Ashton G, Blaylock M, Bagley S, Connolly Y, Bridgeman J, Miller C, Krishnan S, Dempsey $\mathrm{C}$, et al. RAC2, AEP, and ICAM1 expression are associated with CNS disease in a mouse model of pre-B childhood acute lymphoblastic leukemia. Blood. 2011; 118(3):638649. 